\title{
Uso e fontes de obtenção de psicotrópicos em adultos e idosos brasileiros
}

\author{
Use and sources of psychotropic drugs by Brazilian adults and \\ seniors
}

Patrícia Silveira Rodrigues (https://orcid.org/0000-0002-1313-2403) ${ }^{1}$

Priscila Maria Stolses Bergamo Francisco (https://orcid.org/0000-0001-7361-9961) 1,2

Andréia Turmina Fontanella (https://orcid.org/0000-0003-0455-9429) ${ }^{3}$

Rogério Boff Borges (https://orcid.org/0000-0002-2548-1889) ${ }^{3}$

Karen Sarmento Costa (http://orcid.org/0000-0002-2218-6024) ${ }^{1,3}$

${ }^{1}$ Programa de PósGraduação em Saúde Coletiva, Universidade Estadual de Campinas (UNICAMP). R. Tessália Vieira de Camargo 126, Cidade Universitária Zeferino Vaz. 13083-887 Campinas SP Brasil. patsilveirarodrigues@ gmail.com

${ }^{2}$ Faculdade de Ciências Médicas, UNICAMP.

Campinas SP Brasil.

${ }^{3}$ Programa de Pós-

Graduação em

Epidemiologia,

Universidade Federal do Rio

Grande do Sul, Porto Alegre

RS Brasil.

\begin{abstract}
The aim of the present study was to estimate the prevalence of psychotropic drug utilization among adults and seniors and determine associated factors, therapeutic classes and sources of acquisition. Data from the Brazilian National Survey on Access, Use and Promotion of the Rational Use of Medicines (2013-2014) were analyzed. The prevalence of use of at least one psychotropic drug in the overall sample (adults and seniors) was $8.7 \%$. In the adjusted analyses, positive associations were found between the use of psychotropic drugs and the female sex, poorer self-rated health and chronic diseases $(p<0.05)$. The most frequently used therapeutic classes were antidepressants (55.3\%) by the adults and anxiolytics (59.3\%) by the seniors. Approximately $23.0 \%$ of psychotropic drugs were obtained exclusively from pharmacies of the public healthcare system and $77.0 \%$ were acquired from other sources. The findings reveal a low proportion of attaining psychotropic drugs through the Brazilian public healthcare system as well as the need for public policies that encourage the rational use of prescriptions and treatments to promote a better quality of life and ensure the population's right to health.
\end{abstract}

Key words Drug utilization, Psychotropic drugs, Pharmacoepidemiology, Health Surveys
Resumo O objetivo do presente estudo foi verificar a prevalência do uso de psicotrópicos nos adultos e idosos e os fatores associados, classes terapêuticas de medicamentos e fontes de obtenção. Foram analisados dados da Pesquisa Nacional sobre o Acesso, Utilização e Promoção do Uso Racional de Medicamentos no Brasil (PNAUM/2013-2014). A prevalência do uso de ao menos um psicotrópico para o conjunto de adultos brasileiros foi de 8,7\%. Nas análises ajustadas para adultos e idosos, observaram-se associações positivas entre uso de psicotrópicos e sexo feminino, pior autoavaliação de saúde e presença de doenças crônicas $(p<0,05)$. As classes terapêuticas mais utilizadas foram os antidepressivos $(55,3 \%)$ por adultos $e$ os ansiolíticos (59,3\%) por idosos. Cerca de 23,0\% dos psicotrópicos foram obtidos exclusivamente nas farmácias do SUS e os outros $77,0 \%$ por outras fontes. Os resultados mostraram baixa proporção de obtenção dos psicotrópicos no SUS e a necessidade de politicas que incentivem a prescrição $e$ tratamentos com mais racionalidade, promovendo melhor qualidade de vida e garantia do direito à saúde a população.

Palavras-chave Uso de medicamentos, Psicotrópicos, Farmacoepidemiologia, Inquéritos Epidemiológicos 


\section{Introdução}

A transição demográfica e epidemiológica brasileira evidencia os desafios em implementar os princípios constitucionais do Sistema Único de Saúde (SUS), em uma realidade que reúne tripla carga de doenças, com persistência das doenças infecciosas e parasitárias, crescimento das causas externas e das doenças crônicas ${ }^{1}$. Nas últimas décadas, com o acelerado envelhecimento populacional e a elevação da prevalência de doenças crônicas não transmissíveis (DCNT), observa-se uma ampliação da demanda pelo uso de medicamentos por longos períodos ${ }^{2}$.

Os transtornos mentais e comportamentais (TMC) estão entre as DCNT que mais levam a incapacidade e deterioram a qualidade de vida ${ }^{3}$. Evidências do estudo Global Burden of Disease (GBD), apontam que os TMC se traduzem em um grave problema de saúde pública, são altamente prevalentes e representam $18,7 \%$ de todos os anos de vida vividos com incapacidade (YLD) ${ }^{4}$.

Resultados do Estudo GBD no Brasil demonstram que os TMC foram responsáveis por 9,5\% do total dos anos de vida vividos com incapacidade (Disability Adjusted Life Years - DALY) no país. Dentre os transtornos mentais que mais contribuem para os anos de vida saudáveis perdidos estão os transtornos depressivos (3,3\%) e os transtornos de ansiedade (2,7\%). Análises do mesmo estudo, tanto em 1990 quanto em 2015, evidenciam que os TMC foram a principal causa de incapacidade no Brasil, sendo responsáveis por $24,9 \%$ do total de YLD por todas as causas ${ }^{3}$.

$\mathrm{O}$ uso de psicotrópicos tem sido observado massivamente em muitos países, ocupando posição entre as maiores taxas de utilização de medicamentos, com marcada popularização e benefícios inegáveis no tratamento dos $\mathrm{TMC}^{5}$. No entanto, a necessidade dos psicotrópicos como agentes terapêuticos tem sido questionável, principalmente quando a indicação se baseia em quadros psiquiátricos inespecíficos ${ }^{6}$.

O uso extensivo de psicotrópicos reflete uma tendência na recorrência de um fenômeno complexo, polêmico e multifacetado, como o descrito por Birmam ${ }^{7}$, quando retrata a medicalização social. Nesta lógica, os psicotrópicos assumem papel central de transformar os conflitos humanos e o sofrimento psíquico em problemas que se resolvem a partir de uma intervenção medicamentosa.

Dados sobre o acesso e utilização dos psicotrópicos pela população adulta e idosa brasileira ainda não foram descritos na literatura nacional.
Nesse sentido, observa-se uma lacuna quanto ao conhecimento da prevalência de uso de psicotrópicos no país, bem como do perfil dos usuários para melhor compreensão do padrão de uso desses medicamentos. Portanto, este estudo tem como objetivo estimar a prevalência do uso de psicotrópicos na população adulta e idosa, bem como os fatores associados ao uso; estimar a prevalência de uso segundo classes terapêuticas e verificar as fontes de obtenção dos medicamentos segundo região geográfica, classificação econômica e posse de plano de saúde.

\section{Métodos}

Estudo transversal de base populacional, que utilizou dados de indivíduos com idade entre 20 e 59 anos $(n=23.329)$ e idosos com idade $\geq$ 60 anos $(\mathrm{n}=9.019)$ da Pesquisa Nacional sobre Acesso, Utilização e Promoção do Uso Racional de Medicamentos (PNAUM), realizada entre setembro de 2013 e fevereiro de 2014.

O plano amostral da PNAUM componente inquérito foi complexo com amostra probabilística, estratificada por sexo e faixas etárias e realizado em três estágios. Os dados foram coletados por meio questionário estruturado e entrevistas face a face, aplicado a partir de dispositivo eletrônico (tablet) na população residente em área urbana com representação das cinco grandes regiões geográficas do país. O detalhamento dos procedimentos de amostragem e da coleta de dados são descritos por Mengue et al. ${ }^{8}$.

Os dados analisados neste estudo foram obtidos de questionário aplicado a todos os indivíduos adultos (idade $\geq 20$ anos), e que contemplaram informações sobre a utilização de medicamentos para o tratamento de doenças crônicas e/ou eventos agudos relatados por estes entrevistados.

A variável dependente foi o uso de ao menos um medicamento psicotrópico (sim ou não), independentemente do motivo do uso (tratamento de doenças crônicas ou de eventos agudos ocorridos nos 15 dias anteriores à entrevista). As informações sobre o uso de medicamentos foram obtidas por uma sequência de questões que iniciavam a partir da referência do entrevistado, como no exemplo: "Algum médico já lhe disse que o(a) $\operatorname{Sr}(a)$ tem depressão?" (sim/não). Em seguida, perguntava-se: " $\mathrm{O}(a) \mathrm{Sr}(a)$ tem indicação médica para usar algum remédio para a depressão?" ( $\operatorname{sim} /$ não) e: "O(a) Sr(a) está usando algum desses remédios?" (sim/não). Em caso afirmativo: "Quais?" 
$\mathrm{Na}$ análise dos fatores associados ao uso de qualquer psicotrópico, as seguintes variáveis independentes foram selecionadas:

1. Demográficas e socioeconômicas: região de residência (Norte, Nordeste, Sudeste, Sul e Centro-Oeste), sexo (masculino e feminino), cor da pele/raça (branco e não branco), situação conjugal (com e sem cônjuge), escolaridade (0 a 8 anos, 9 a 11 anos, 12 e mais anos), classificação econômica (A/B; C; D; E), segundo o Critério de Classificação Econômica Brasil desenvolvido pela Associação Brasileira de Empresas de Pesquisa (CCEB 2013/ABEP - http://www.abep.org/) e posse de plano de saúde (sim ou não). Quanto a classificação econômica, os indivíduos pertencentes a classe A corresponderam a um pequeno percentual $(0,5 \%)$ e, dada a similaridade entre os perfis de consumo e poder de compra das pessoas e famílias das classes A e B, optou-se por agregá -las em uma única categoria.

2. Comportamentos e condições de saúde: autoavaliação de saúde (muito boa/boa, regular e ruim/muito ruim), número de doenças crônicas (nenhuma, uma, duas e mais), tabagismo (sim ou não) e consumo abusivo de bebidas alcoólicas (quatro ou mais doses para mulher e cinco ou mais doses para homem em uma mesma ocasião nos últimos 30 dias, considerando como dose de bebida alcoólica uma dose de bebida destilada, uma lata de cerveja ou uma taça de vinho). Para compor a variável número de doenças crônicas foram consideradas as seguintes doenças referidas pelo entrevistado: hipertensão arterial, diabetes mellitus, doença respiratória pulmonar, colesterol alto, acidente vascular cerebral, doenças do coração, doença articular crônica e outras doenças crônicas (sim ou não).

Os psicotrópicos foram classificados em quatro classes terapêuticas de acordo com o European Study of the Epidemiology of Mental Disorders (ESEMeD): fármacos antidepressivos, ansiolíticos, antipsicóticos e os medicamentos estabilizadores de humor.

Quanto à fonte de obtenção de medicamentos para tratamento das suas condições clínicas, os entrevistados foram inqueridos sobre os locais de obtenção e, para os que referiram o uso de psicotrópicos, criou-se uma variável dicotômica que indicava a obtenção exclusiva de todos os psicotrópicos utilizados nas farmácias do Sistema Único de Saúde (SUS-exclusivo) ou não (exclusivamente na Farmácia Popular; exclusivamente na Farmácia Privada; no SUS e na Farmácia Popular; no SUS e em Farmácia Privada; Farmácia Popular e Farmácia Privada; SUS, Farmácia Popular e Farmácia Privada).
Inicialmente foram estimadas as prevalências de uso de psicotrópicos entre adultos e idosos, segundo as variáveis selecionadas. A associação entre as diversas variáveis e o uso de ao menos um psicotrópico, foi verificada pelo teste quiquadrado de Pearson com correção de segunda ordem proposta por Rao e Scott para amostras complexas, considerando-se um nível de significância de 5\%.

As variáveis independentemente associadas ao uso foram identificadas por meio de regressão múltipla de Poisson em que o critério de inclusão das variáveis no modelo foi apresentar associação em nível menor que 0,20 na análise bivariada. Em relação às doenças referidas, no modelo múltiplo foi considerada apenas a variável número de doenças crônicas. A entrada das variáveis no modelo múltiplo seguiu o procedimento de seleção backward e permaneceram no modelo apenas aquelas variáveis com $p<0,05$. Também foram estimadas as prevalências de uso de psicotrópicos na população adulta e idosa segundo classe terapêutica e os respectivos intervalos de confiança de $95 \%$.

Por fim, verificaram-se as fontes de obtenção de medicamentos utilizadas pelos entrevistados que referiram uso de psicotrópicos (exclusivamente no SUS ou não), segundo região de residência, classificação econômica e plano de saúde. As análises foram realizadas Stata 14.0 considerando-se as ponderações decorrentes do delineamento amostral complexo da pesquisa.

A PNAUM foi aprovada pela Comissão $\mathrm{Na}$ cional de Ética em Pesquisa e pelo Comitê de Ética em Pesquisa da Universidade Federal do Rio Grande do Sul. Todos os participantes assinaram termo de consentimento livre e esclarecido.

\section{Resultados}

Neste estudo a média de idade foi de 39,6 anos $(\mathrm{dp}=11,2)$ e de $70,2(\mathrm{dp}=8,0)$ nos adultos e idosos, respectivamente. A prevalência geral de uso de psicotrópicos na população adulta e idosa ( $\geq 20$ anos) foi de 8,7\% (IC95\%: 8,1-9,4), com diferença significativa entre os indivíduos ( $\mathrm{p}$ $<0,001)$ com idade entre 20 e 59 anos $(7,0 \%$; IC95\%:6,4-7,7) e naqueles com idade $\geq 60$ anos (16,1\%; IC95\%:14,7-17,5).

Na Tabela 1 é apresentada a distribuição da população e a prevalência de uso de pelo menos um psicotrópico, segundo características sociodemográficas. Destaca-se que $76,4 \%$ dos indivíduos com idade $\geq 20$ anos não possuíam plano de saúde à época da pesquisa. Para ambos os grupos 
Tabela 1. Distribuição da amostra e prevalência de uso de pelo menos um psicotrópico, segundo características sociodemográficas na população adulta e idosa. Pesquisa Nacional sobre o Acesso, Utilização e Promoção do Uso Racional de Medicamentos no Brasil (PNAUM), Brasil, 2014.

\begin{tabular}{|c|c|c|c|c|c|c|c|c|}
\hline \multirow{2}{*}{ Variáveis } & \multirow{2}{*}{$\mathbf{n}$} & \multirow{2}{*}{$(\%)$} & \multicolumn{2}{|c|}{ Total } & \multicolumn{2}{|c|}{ Adultos } & \multicolumn{2}{|c|}{ Idosos } \\
\hline & & & $\%$ & IC95\% & $\%$ & IC95\% & $\%$ & IC95\% \\
\hline Região de residência & & & \multicolumn{2}{|c|}{$\mathrm{p}^{\star}<0,001$} & \multicolumn{2}{|c|}{$\mathrm{p}<0,001$} & \multicolumn{2}{|c|}{$\mathrm{p}<0,001$} \\
\hline Norte & 8.421 & 6,7 & 2,1 & $1,6-2,7$ & 1,9 & $1,4-2,5$ & 3,2 & $2,5-4,2$ \\
\hline Nordeste & 6.909 & 23,4 & 7,5 & $6,7-8,5$ & 6,5 & $5,7-7,3$ & 12,3 & $10,8-14,1$ \\
\hline Sudeste & 6.075 & 47,4 & 9,6 & $8,5-10,9$ & 7,6 & $6,5-8,8$ & 17,3 & $15,1-19,8$ \\
\hline Sul & 6.097 & 14,7 & 11,4 & $10,2-12,6$ & 8,8 & $7,7-10,0$ & 22,3 & $19,8-25,0$ \\
\hline Centro-Oeste & 4.846 & 7,8 & 7,6 & $6,6-8,8$ & 6,5 & $5,4-7,7$ & 13,7 & $11,8-15,8$ \\
\hline Sexo & & & \multicolumn{2}{|c|}{$\mathrm{p}<\mathbf{0 , 0 0 1}$} & \multicolumn{2}{|c|}{$\mathrm{p}<\mathbf{0 , 0 0 1}$} & \multicolumn{2}{|c|}{$\mathbf{p}<\mathbf{0 , 0 0 1}$} \\
\hline Masculino & 11.702 & 46,3 & 5,1 & $4,5-5,7$ & 3,9 & $3,4-4,6$ & 10,4 & $9,1-11,9$ \\
\hline Feminino & 20.646 & 53,7 & 11,9 & $10,9-12,9$ & 9,8 & $8,8-10,8$ & 20,2 & $18,4-22,2$ \\
\hline Cor da pele/raça & & & \multicolumn{2}{|c|}{$\mathrm{p}<\mathbf{0 , 0 0 1}$} & \multicolumn{2}{|c|}{$\mathbf{p}<\mathbf{0 , 0 0 1}$} & \multicolumn{2}{|c|}{$\mathbf{p}<\mathbf{0 , 0 0 1}$} \\
\hline Brancos & 13.890 & 48,0 & 10,7 & $9,8-11,6$ & 8,4 & $7,5-9,4$ & 19,0 & $17,4-20,7$ \\
\hline Não brancos & 16.725 & 52,0 & 7,7 & $6,9-8,5$ & 6,4 & $5,7-7,3$ & 13,7 & $11,8-15,8$ \\
\hline Situação conjugal & & & \multicolumn{2}{|c|}{$\mathrm{p}<\mathbf{0 , 0 0 1}$} & \multicolumn{2}{|c|}{$\mathrm{p}=\mathbf{0 , 0 3 3}$} & \multicolumn{2}{|c|}{$\mathrm{p}<\mathbf{0 , 0 0 1}$} \\
\hline Com companheiro & 19.647 & 60,7 & 8,2 & $7,5-9,0$ & 6,9 & $6,1-7,7$ & 14,5 & $13,0-16,1$ \\
\hline Sem companheiro & 11.586 & 39,3 & 10,4 & $9,5-11,5$ & 8,1 & $7,2-9,1$ & 18,9 & $16,8-21,2$ \\
\hline Escolaridade & & & \multicolumn{2}{|c|}{$\mathrm{p}=\mathbf{0 , 0 8 3}$} & \multicolumn{2}{|c|}{$p=0,200$} & \multicolumn{2}{|c|}{$\mathrm{p}=0,302$} \\
\hline 0 a 8 & 18.536 & 58,3 & 9,2 & $8,4-10,0$ & 7,4 & $6,6-8,3$ & 16,7 & $15,2-18,3$ \\
\hline 9 a 11 & 9.997 & 30,6 & 8,0 & $7,1-9,0$ & 6,4 & $5,5-7,3$ & 14,9 & $12,6-17,6$ \\
\hline 12 e mais & 3.602 & 11,1 & 8,2 & $6,9-10,0$ & 6,7 & $5,4-8,2$ & 15,1 & $12,4-18,4$ \\
\hline Classificação econômica & & & \multicolumn{2}{|c|}{$\mathrm{p}=0,1129$} & \multicolumn{2}{|c|}{$\mathrm{p}=\mathbf{0 , 0 7 5}$} & \multicolumn{2}{|c|}{$\mathrm{p}=\mathbf{0 , 8 9 5}$} \\
\hline $\mathrm{A} / \mathrm{B}$ & 6.774 & 24,2 & 8,6 & $7,5-10,0$ & 7,1 & $5,9-8,6$ & 16,0 & $13,4-18,9$ \\
\hline $\mathrm{C}$ & 18.209 & 55,1 & 8,3 & $7,5-9,1$ & 6,4 & $5,7-7,2$ & 16,3 & $14,7-18,0$ \\
\hline $\mathrm{D}$ & 5.940 & 16,2 & 9,8 & $8,5-11,3$ & 8,3 & $6,9-10,0$ & 15,2 & $12,7-18,0$ \\
\hline $\mathrm{E}$ & 1.366 & 4,5 & 10,2 & $8,1-12$ & 8,8 & $6,4-12,0$ & 16,1 & $11,9-21,4$ \\
\hline Plano de saúde & & & \multicolumn{2}{|c|}{$\mathrm{p}<0,001$} & \multicolumn{2}{|c|}{$\mathrm{p}=0,054$} & \multicolumn{2}{|c|}{$\mathrm{p}<0,001$} \\
\hline Sim & 6.156 & 23,6 & 10,8 & $9,4-12,3$ & 8,2 & $6,8-9,7$ & 20,2 & $17,6-23,1$ \\
\hline Não & 26.156 & 76,4 & 8,1 & $7,4-8,8$ & 6,7 & $6,0-7,4$ & 14,5 & $13,2-16,0$ \\
\hline
\end{tabular}

etários, observam-se diferenças nas prevalências de acordo com a região de residência, sexo, cor da pele e situação conjugal $(p<0,05)$. Para os idosos, também foi observada maior prevalência entre os que referiram posse de plano de saúde ( $\mathrm{p}$ $<0,001)$. Para todas as variáveis, observaram-se maiores prevalências de uso nos idosos.

A prevalência de uso de ao menos um psicotrópico, segundo comportamentos e condições de saúde na população adulta e idosa, é apresentada na Tabela 2. Na população adulta, todas as variáveis associaram-se ao uso $(\mathrm{p}<0,01)$. Nos idosos, apenas o tabagismo $(\mathrm{p}=0,92)$ não apresentou associação ao uso de psicotrópicos.
Os resultados finais da análise múltipla dos fatores associados ao uso de psicotrópicos são apresentados na Tabela 3. Tanto para os adultos quando para os idosos, maiores prevalências de uso foram observadas nas regiões Nordeste, Sudeste, Sul e Centro-Oeste em relação à região Norte. Também foram significativamente mais elevadas entre as mulheres, nos que avaliaram sua saúde como regular ou ruim/muito ruim, naqueles que referiram ao menos uma doença crônica e nos fumantes. Particularmente nos adultos, indivíduos com classificação econômica "E" também apresentaram maior prevalência de uso (RP=1,41; IC95\%:1,01-1,98) e menores 
Tabela 2. Prevalência de uso de ao menos um psicotrópico, segundo comportamentos e condições de saúde na população adulta e idosa. Pesquisa Nacional sobre o Acesso, Utilização e Promoção do Uso Racional de Medicamentos no Brasil (PNAUM), Brasil, 2014.

\begin{tabular}{|c|c|c|c|c|c|c|}
\hline \multirow{2}{*}{ Variáveis } & \multicolumn{2}{|c|}{ Total } & \multicolumn{2}{|c|}{ Adultos } & \multicolumn{2}{|c|}{ Idosos } \\
\hline & $\%$ & IC95\% & $\%$ & IC95\% & $\%$ & IC95\% \\
\hline Tabagismo & \multicolumn{2}{|c|}{$\mathrm{p}^{\star}=0,0152$} & \multicolumn{2}{|c|}{$\mathrm{p}<0,001$} & \multicolumn{2}{|c|}{$p=0,921$} \\
\hline Não & 7,8 & $7,2-8,5$ & 6,1 & $5,5-6,8$ & 15,2 & $13,8-16,8$ \\
\hline Sim & 9,5 & $8,2-11,0$ & 8,6 & $7,1-10,2$ & 15,0 & $12,2-18,4$ \\
\hline Consumo abusivo de álcool & \multicolumn{2}{|c|}{$\mathbf{p}<0,001$} & \multicolumn{2}{|c|}{$\mathrm{p}<0,001$} & \multicolumn{2}{|c|}{$\mathbf{p}<0,001$} \\
\hline Não & 9,6 & $8,9-10,3$ & 7,8 & $7,2-8,5$ & 16,5 & $15,1-17,9$ \\
\hline Sim & 2,3 & $1,6-3,3$ & 2,1 & $1,3-11,2$ & 6,4 & $3,5-11,2$ \\
\hline Autoavaliação da saúde & \multicolumn{2}{|c|}{$\mathbf{p}<0,001$} & \multicolumn{2}{|c|}{$\mathrm{p}<0,001$} & \multicolumn{2}{|c|}{$\mathbf{p}<\mathbf{0 , 0 0 1}$} \\
\hline Muito boa/boa & 5,0 & $4,5-5,5$ & 4,0 & $3,5-4,5$ & 10,8 & $9,5-12,3$ \\
\hline Regular & 16,8 & $15,2-18,3$ & 15,1 & $13,4-17,0$ & 20,4 & $18,2-22,7$ \\
\hline Ruim/muito ruim & 35,5 & $31,6-39,6$ & 34,9 & $30,1-40,0$ & 36,6 & $30,9-42,7$ \\
\hline Número de DCNT & \multicolumn{2}{|c|}{$\mathrm{p}<0,001$} & \multicolumn{2}{|c|}{$\mathrm{p}<\mathbf{0 , 0 0 1}$} & \multicolumn{2}{|c|}{$\mathbf{p}<\mathbf{0 , 0 1}$} \\
\hline Nenhuma & 3,3 & $2,8-3,8$ & 3,0 & $2,5-3,4$ & 7,4 & $6,0-9,2$ \\
\hline Uma & 13,8 & $12,6-15,1$ & 14,4 & $12,9-16,1$ & 12,2 & $10,7-14,0$ \\
\hline Duas e mais & 22,0 & $31,6-39,6$ & 21,0 & $18,4-24,0$ & 22,9 & $20,6-25,4$ \\
\hline \multicolumn{7}{|l|}{ Doenças referidas } \\
\hline Hipertensão arterial & \multicolumn{2}{|c|}{$\mathrm{p}<\mathbf{0 , 0 0 1}$} & \multicolumn{2}{|c|}{$\mathrm{p}<0,001$} & \multicolumn{2}{|c|}{$\mathrm{p}<\mathbf{0 , 0 0 1}$} \\
\hline Não & 6,1 & $5,6-6,7$ & 5,5 & $5,0-6,1$ & 11,9 & $10,3-13,5$ \\
\hline Sim & 16,8 & $15,4-18,4$ & 15,0 & $13,2-16,9$ & 19,0 & $17,3-20,9$ \\
\hline Diabetes mellitus & \multicolumn{2}{|c|}{$\mathrm{p}<0,001$} & \multicolumn{2}{|c|}{$\mathrm{p}<0,001$} & \multicolumn{2}{|c|}{$\mathrm{p}=0,015$} \\
\hline Não & 8,0 & $7,4-8,6$ & 6,6 & $6,0-7,2$ & 15,5 & $14,2-16,9$ \\
\hline Sim & 18,0 & $15,9-20,4$ & 17,4 & $14,4-20,9$ & 18,6 & $15,9-21,5$ \\
\hline Doença respiratória pulmonar & \multicolumn{2}{|c|}{$\mathrm{p}<0,001$} & \multicolumn{2}{|c|}{$\mathbf{p}<0,001$} & \multicolumn{2}{|c|}{$\mathrm{p}=\mathbf{0 , 0 0 1}$} \\
\hline Não & 8,3 & $7,7-9,0$ & 6,7 & $6,1-7,3$ & 15,7 & $14,4-17,2$ \\
\hline Sim & 19,6 & $16,1-23,6$ & 18,2 & $14,0-23,3$ & 22,9 & $18,2-28,3$ \\
\hline Colesterol alto & \multicolumn{2}{|c|}{$\mathrm{p}<0,001$} & &, 001 & &, 001 \\
\hline Não & 7,4 & $6,8-8,1$ & 6,2 & $5,7-6,9$ & 13,6 & $12,4-14,9$ \\
\hline Sim & 20,2 & $17,8-22,8$ & 17,1 & $14,2-20,4$ & 24,3 & $21,1-27,9$ \\
\hline Acidente vascular cerebral & & 001 & &, 001 & &, 001 \\
\hline Não & 8,4 & $7,8-9,1$ & 6,9 & $6,3-7,5$ & 15,5 & $14,1-16,9$ \\
\hline Sim & 26,4 & $21,7-31,8$ & 27,4 & $19,6-36,9$ & 25,8 & $20,9-31,5$ \\
\hline Doenças do coração & & 001 & &, 001 & & ,001 \\
\hline Não & 7,9 & $7,3-8,6$ & 6,6 & $6,0-7,3$ & 14,6 & $13,3-16,0$ \\
\hline Sim & 23,2 & $20,4-26,3$ & 21,7 & $17,8-26,1$ & 24,5 & $20,9-28,4$ \\
\hline Doença articular crônica & & 001 & &, 001 & &, 001 \\
\hline Não & 7,6 & $7,0-8,3$ & 6,4 & $5,8-2,7$ & 13,7 & $12,3-15,1$ \\
\hline Sim & 25,6 & $23,0-28,4$ & 22,7 & $18,6-27,5$ & 28,3 & $24,9-31,9$ \\
\hline
\end{tabular}

*Valor de $\mathrm{p}$ do teste qui-quadrado (Rao-Scott).

prevalências foram observadas em não brancos $(\mathrm{RP}=0,78$; IC95\%: 0,67-0,91). Nos idosos verificou-se menor prevalência de uso de psicotrópicos naqueles que não possuíam plano de saúde à época da pesquisa $(\mathrm{RP}=0,81$; IC95\%: 0,70-0,93).

Dentre os indivíduos que referiram uso de psicotrópicos, na avaliação segundo classes terapêuticas, $55,3 \%$ dos adultos e $49,6 \%$ dos idosos referiram o uso de antidepressivos. Os ansiolíticos também apresentaram elevada proporção de uso, principalmente nos idosos (59,3\%) (Figura 1).

Na Tabela 4, observa-se que, para o conjunto dos adultos ( $\geq 20$ anos), a obtenção de psicotrópicos exclusivamente no SUS variou de 20,1\% na região Centro-Oeste a $36,2 \%$ na região Norte, 
Tabela 3. Modelo de regressão múltipla de Poisson para o uso de ao menos um psicotrópico na população adulta e idosa. Pesquisa Nacional sobre o Acesso, Utilização e Promoção do Uso Racional de Medicamentos no Brasil (PNAUM), Brasil, 2014.

\begin{tabular}{|c|c|c|c|c|}
\hline \multirow{2}{*}{ Variáveis/categorias } & \multicolumn{2}{|c|}{ Adultos } & \multicolumn{2}{|c|}{ Idosos } \\
\hline & RP & IC95\% & RP & IC95\% \\
\hline \multicolumn{5}{|l|}{ Região de residência } \\
\hline Norte & 1 & & 1 & \\
\hline Nordeste & 2,63 & $1,94-3,57$ & 3,17 & $2,35-4,28$ \\
\hline Sudeste & 3,80 & $2,76-5,22$ & 4,82 & $3,56-6,54$ \\
\hline Sul & 3,99 & $2,93-5,43$ & 6,03 & $4,50-8,08$ \\
\hline Centro-Oeste & 2,95 & $2,14-4,06$ & 3,51 & $2,60-4,75$ \\
\hline \multicolumn{5}{|l|}{ Sexo } \\
\hline Masculino & 1 & & 1 & \\
\hline Feminino & 1,92 & $1,61-2,31$ & 1,69 & $1,47-1,94$ \\
\hline \multicolumn{5}{|l|}{ Cor da pele/raça } \\
\hline Brancos & 1 & & & \\
\hline Não brancos & 0,78 & $0,67-0,91$ & & \\
\hline \multicolumn{5}{|l|}{ Classificação econômica } \\
\hline $\mathrm{A} / \mathrm{B}$ & 1 & & & \\
\hline $\mathrm{C}$ & 0,91 & $0,7-1,12$ & & \\
\hline $\mathrm{D}$ & 1,05 & $0,81-1,37$ & & \\
\hline $\mathrm{E}$ & 1,41 & $1,01-1,98$ & & \\
\hline \multicolumn{5}{|l|}{ Plano de saúde } \\
\hline $\operatorname{Sim}$ & & & 1 & \\
\hline Não & & & 0,81 & $0,70-0,93$ \\
\hline \multicolumn{5}{|l|}{ Autoavaliação da saúde } \\
\hline Muito boa/boa & 1 & & 1 & \\
\hline Regular & 2,35 & $2,02-2,73$ & 1,64 & $1,42-1,90$ \\
\hline Ruim/muito ruim & 4,44 & $3,52-5,59$ & 2,84 & $2,32-3,44$ \\
\hline \multicolumn{5}{|l|}{ Número de DCNT } \\
\hline Nenhuma & 1 & & 1 & \\
\hline Uma & 3,08 & $2,54-3,73$ & 1,35 & $1,06-1,72$ \\
\hline Duas e mais & 3,47 & $2,73-4,40$ & 1,92 & $1,51-2,45$ \\
\hline \multicolumn{5}{|l|}{ Tabagismo } \\
\hline Não & 1 & & & \\
\hline $\operatorname{Sim}$ & 1,24 & $1,04-1,49$ & & \\
\hline \multicolumn{5}{|l|}{ Consumo abusivo de álcool } \\
\hline Não & 1 & & & \\
\hline Sim & 0,42 & $0,28-0,66$ & & \\
\hline
\end{tabular}

sem diferenças entre as regiões brasileiras. Também foi verificada maior obtenção entre os que declararam não possuir plano de saúde e, quanto à classe econômica, o percentual foi cerca de duas vezes maior nas classes $\mathrm{C}, \mathrm{D}$ e $\mathrm{E}$ em relação às classes A/B.

Considerando-se apenas os adultos, observaram-se diferenças nos percentuais de obtenção quanto à classe econômica e ao plano de saúde. Já para os idosos, houve diferenças na obtenção em relação à região de residência, variando de 11,0\% na região Nordeste a 23,9\% no Sudeste. Nos idosos sem plano de saúde, a obtenção de psicotrópicos foi significativamente maior (Tabela 4).

\section{Discussão}

Os resultados desta pesquisa apontam, tanto para os adultos quanto para os idosos, que a maior 
Estabilizadores de humor

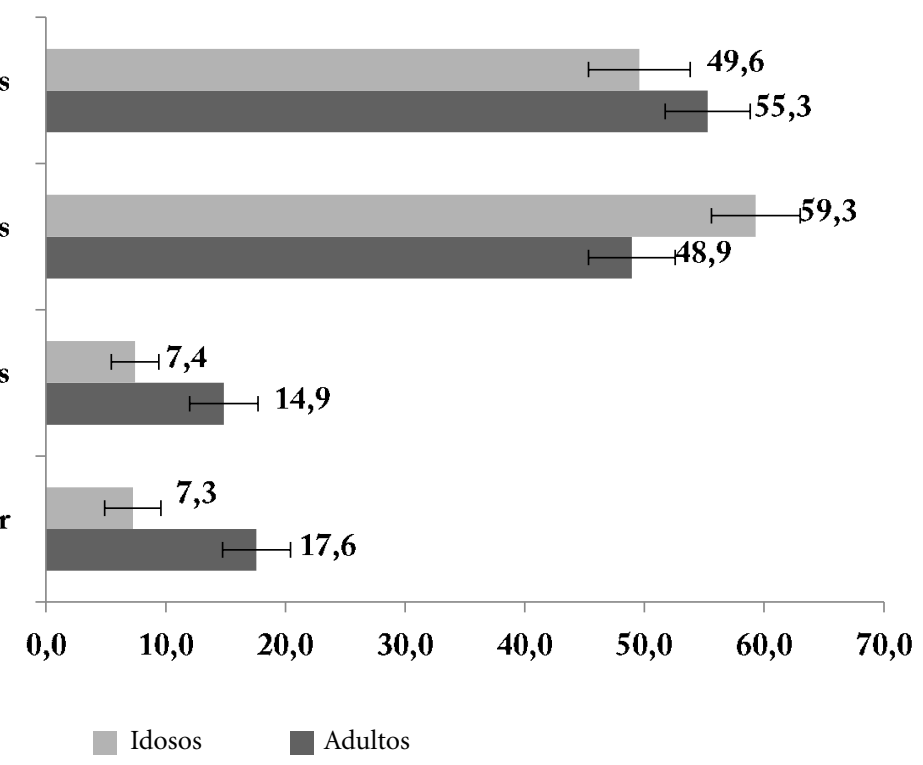

Figura 1. Psicotrópicos segundo classes terapêuticas, de acordo com o European Study of the Epidemiology of Mental Disorders (ESEMeD). Pesquisa Nacional sobre o Acesso, Utilização e Promoção do Uso Racional de Medicamentos no Brasil (PNAUM), Brasil, 2014.

utilização de psicotrópicos foi observada entre mulheres, dentre os que referiram autopercepção negativa de saúde e naqueles indivíduos com o maior número de doenças crônicas. Menor prevalência de uso foi encontrada nas regiões Norte e Nordeste do Brasil.

A prevalência do uso de psicotrópicos observada na população adulta e idosa brasileira residente na zona urbana foi de $8,7 \%$. A ausência de estudos nacionais anteriores, quanto à utilização de psicotrópicos na população adulta e idosa do Brasil, impossibilitam comparações com dados de pesquisas anteriores, bem como o conhecimento do perfil de utilização desses medicamentos pela população.

Estudos realizados em outros países como Canadá $(7,2 \%)^{9}$ e Estados Unidos $(11,1 \%)^{10}$ demonstram prevalências da utilização de psicotrópicos similares à encontrada no Brasil, porém, superiores àquelas de países do ocidente europeu (França, Alemanha, Itália e Reino Unido) ${ }^{11}$ e Chi$\mathrm{l}^{12}, \mathrm{e}$ inferior à prevalência observada na população espanhola $(16 \%)^{13}$. Tais informações retratam um panorama geral do uso de psicotrópicos em outros países, no entanto, as comparações entre essas prevalências exigem cautela. Diferenças observadas em estudos de utilização de medi- camentos podem estar relacionadas aos métodos usados pela pesquisa, que incluem a população de estudo, o período recordatório, distinção entre faixas etárias e tipos de medicamentos investigados, entre outros. Além disso, discrepâncias nos Sistemas de Saúde ao redor do mundo, relacionadas ao acesso a serviços de saúde e a oferta e prescrição de medicamentos, assim como o contexto do período em que o estudo foi realizado, podem influenciar na magnitude da frequência de uso dos psicotrópicos.

O consumo de psicotrópicos foi significativamente superior nas mulheres. Vários estudos constatam essa associação com o sexo feminino ${ }^{13,14}$. Tradicionalmente, mulheres buscam mais ajuda para o autocuidado e usam com mais frequência os serviços de saúde ${ }^{15}$. $\mathrm{O}$ histórico da organização dos serviços médicos e construção dos programas de saúde no Brasil tiveram como foco central a saúde materno-infantil e a saúde reprodutiva da mulher, fortalecendo o processo de medicalização social do corpo feminino ${ }^{16}$. Mulheres têm maior carga para os transtornos depressivos e de ansiedade, e maior ocorrência de transtornos alimentares e de personalidade boderline $\mathrm{e}^{17}$.

A diferença entre os sexos no processo de medicalização é fortalecida pelo papel social im- 
Tabela 4. Fontes de obtenção de psicotrópicos utilizados por adultos e idosos, de acordo com a região do país, classificação econômica e plano de saúde. Pesquisa Nacional sobre o Acesso,

Utilização e Promoção do Uso Racional de Medicamentos no Brasil (PNAUM), Brasil, 2014.

\begin{tabular}{|c|c|c|c|c|c|c|c|c|c|c|c|c|}
\hline \multirow{3}{*}{ Variáveis } & \multicolumn{4}{|c|}{ Total } & \multicolumn{4}{|c|}{ Adultos } & \multicolumn{4}{|c|}{ Idosos } \\
\hline & \multicolumn{2}{|c|}{ SUS-exclusivo } & \multicolumn{2}{|c|}{ Outras } & \multicolumn{2}{|c|}{ SUS-exclusivo } & \multicolumn{2}{|c|}{ Outras } & \multicolumn{2}{|c|}{ SUS-exclusivo } & \multicolumn{2}{|c|}{ Outras } \\
\hline & $\%$ & IC95\% & $\%$ & IC95\% & $\%$ & IC95\% & $\%$ & IC95\% & $\%$ & IC95\% & $\%$ & IC95\% \\
\hline Região de residência & \multicolumn{4}{|c|}{$\mathrm{p}=0,079$} & \multicolumn{4}{|c|}{$p=0,1611$} & \multicolumn{4}{|c|}{$p=0,0045$} \\
\hline Norte & 36,2 & $24,9-49,3$ & 63,8 & $50,7-75,1$ & 40,7 & $27,5-55,4$ & 59,3 & $44,6-72,5$ & 19,8 & $12,2-30,4$ & 80,2 & $69,6-87,8$ \\
\hline Nordeste & 21,4 & $16,9-26,6$ & 78,6 & $73,4-83,1$ & 25,4 & $19,6-32,3$ & 74,6 & $67,7-80,4$ & ] 11 & $7,7-15,5$ & 89 & $84,5-92,3$ \\
\hline Sudeste & 28,4 & $23,1-34,4$ & 71,6 & $65,6-76,9$ & 31,2 & $24,5-38,5$ & 68,8 & $61,5-75,2$ & 23,9 & $18,3-30,5$ & 76,1 & $69,5-81,7$ \\
\hline Sul & 26,9 & $22,2-32,1$ & 73,1 & $67,9-77,8$ & 31,5 & $25,6-38,1$ & 68,5 & $61,9-74,4$ & 18,8 & $14,4-24,2$ & 81,2 & $75,8-85,5$ \\
\hline Centro-Oeste & 20,1 & $15,6-25,5$ & 79,9 & $74,5-84,4$ & 20,7 & $15,1-27,8$ & 79,3 & $72,2-84,9$ & 18,5 & $12,3-27,0$ & 81,5 & $73,0-87,7$ \\
\hline Classificação econômica & \multicolumn{4}{|c|}{$\mathrm{p}<\mathbf{0 , 0 0 1}$} & \multicolumn{4}{|c|}{$\mathbf{p}<\mathbf{0 , 0 0 1}$} & \multicolumn{4}{|c|}{$\mathrm{p}=0,2418$} \\
\hline $\mathrm{A} / \mathrm{B}$ & 14,4 & $10,7-19,1$ & 85,6 & $80,3-90,6$ & 13,7 & $9,4-19,7$ & 86,3 & $80,3-90,6$ & 15,9 & $10,3-23,8$ & 84,1 & $76,2-89,7$ \\
\hline $\mathrm{C}$ & 29,1 & $25,3-33,1$ & 70,9 & $66,9-74,7$ & 33,3 & $28,5-38,4$ & 66,7 & $61,5-71,5$ & 21,8 & $17,7-26,6$ & 78,2 & $73,4-82,3$ \\
\hline $\mathrm{D}$ & 30,5 & $24,5-37,3$ & 69,5 & $62,7-75,5$ & 37 & $28,9-45,8$ & 63 & $54,2-71,0$ & 18,2 & $12,4-26,0$ & 81,8 & $74,0-87,6$ \\
\hline $\mathrm{E}$ & 35,1 & $22,8-49,7$ & 64,9 & $50,3-77,2$ & 37,8 & $22,1-56,5$ & 62,2 & $43,5-77,9$ & 29,5 & $15,4-49,1$ & 70,5 & $50,9-84,6$ \\
\hline Plano de saúde & \multicolumn{4}{|c|}{$\mathbf{p}<\mathbf{0 , 0 0 1}$} & \multicolumn{4}{|c|}{$\mathbf{p}<\mathbf{0 , 0 0 1}$} & \multicolumn{4}{|c|}{$\mathbf{p}<\mathbf{0 , 0 0 1}$} \\
\hline Sim & 10,1 & $7,3-13,8$ & 89,9 & $86,1-92,7$ & 9,3 & $6,0-14,1$ & 90,7 & $85,8-94,0$ & 11,2 & $7,5-16,5$ & 88,8 & $83,4-92,5$ \\
\hline Não & 32,8 & $29,3-36,6$ & 67,2 & $63,4-70,7$ & 36,5 & $32,2-41,0$ & 63,5 & $59,0-67,8$ & 25,2 & $21,2-29,7$ & 74,8 & $70,3-78,8$ \\
\hline Total & 23 & $21,1-25,1$ & 77 & $74,9-78,9$ & 21,5 & $19,6-23,6$ & 78,5 & $76,3-80,4$ & 20,4 & $16,7-24,4$ & 79,6 & $75,6-83,1$ \\
\hline
\end{tabular}


posto às mulheres na sociedade, como o de cuidadora do núcleo familiar e gestora dos negócios do lar, gerando cansaço exaustivo e acúmulo de atribuições e papéis. Estes contextos favorecem a produção de vulnerabilidades e sofrimento, tornando-as alvo de diagnósticos com indicação de tratamentos com produtos farmacêuticos. Também deve-se considerar que não é raro o uso de psicotrópicos, incluindo ansiolíticos e antidepressivos, prescritos pelas mais diversas especialidades médicas, para o tratamento de problemas osteoarticulares, outras dores crônicas, insônia, hipertensão e para o emagrecimento ${ }^{18}$, o que corrobora para o alto consumo desses medicamentos em mulheres.

O crescente consumo de medicamentos psicotrópicos com o avançar da idade (maior entre os idosos), independente do sexo, é registrado em outros estudos nacionais e internacionais , $^{9,12,14,19-21}$. A prevalência no uso de psicotrópicos foi maior naqueles com idade acima dos 60 anos $(16,1 \%)$ e mais elevada se comparada ao resultado do estudo transversal de base populacional realizado no município de Campinas $(10,8 \%)^{14}$. Os achados apontam que a maior utilização nos idosos está associada ao sexo feminino, pior autoavaliação de saúde e maior número de doenças crônicas, fatores relacionados com a senilidade. Ainda, deve-se considerar que, eventualmente, a redução do uso de álcool e outras substâncias psicoativas com o avançar da idade, pode estar associado ao aumento no consumo de psicotrópicos ${ }^{20}$.

Neste estudo, os adultos não brancos utilizam psicotrópicos em menor proporção, o que não foi observado para os idosos. Estudos de base populacional realizados em Pelotas com a população urbana com idade de 15 anos ou mais ${ }^{22} \mathrm{e}$ em Campinas com população indivíduos adultos (idade $\geq 20$ anos) $^{14}$ encontraram resultados semelhantes. A diferença no consumo de psicotrópicos segundo raça/cor da pele tem sido atribuída as iniquidades sociais no acesso aos serviços de saúde e ao tratamento ${ }^{23,24}$. Ainda, o processo de construção social do estigma da doença mental dificulta o tratamento nos indivíduos não brancos, já tão expostos a situações de discriminação e racismo durante o ciclo de vida ${ }^{25}$.

Identificou-se diferença entre as regiões do Brasil no uso de psicotrópicos. A região Norte apresentou a menor prevalência no uso referido desses medicamentos em relação as demais regiões. Na região Sul, a prevalência encontrada foi cerca de 4 vezes maior do que a observada no Norte. Essa diferença evidencia a profunda desigualdade no acesso aos serviços de saúde, diagnósti- co e tratamento da população brasileira, já que a prescrição do psicotrópico é realizada a partir de diagnóstico preliminar ${ }^{26}$ e conta com regulamentação específica para obtenção ${ }^{27}$. Estudo realizado a partir dos dados da Pesquisa Nacional de Amostra de Domicílios (PNAD) dos anos de 1998 e 2008, confirma que dependendo da região de residência há maior ou menor dificuldade em acessar serviços de saúde ${ }^{28}$. Residentes das regiões Norte e Nordeste têm maior dificuldade no acesso aos serviços de saúde, sobretudo na região Norte, onde a configuração geográfica estabelece numerosos desafios à implementação dos serviços ações de saúde e fixação de profissionais em áreas remotas, que dependem de uma complexa malha fluvial ${ }^{29}$.

Deve-se considerar que no caso do uso de psicotrópicos, alguns fatores como o reconhecimento social dos TMC e a organização de um sistema de atenção à saúde com ações e serviços aptos a diagnosticar a doença e ofertar opções terapêuticas, incluindo um sistema adequado de fornecimentos e distribuição de medicamentos, podem impactar no acesso aos psicotrópicos para o tratamento dos problemas de saúde.

Os achados dessa pesquisa corroboram com outros estudos que abordam a autopercepção negativa de saúde como um fator preditor para o consumo de psicotrópicos ${ }^{13,14}$. Um dos pressupostos para este fato é a estreita relação entre multimorbidades crônicas, autopercepção negativa de saúde e aumento no consumo de medicamentos, incluindo os psicotrópicos. Além disso, deve-se considerar que os TMC geram incapacidades que podem refletir na autopercepção da saúde. A associação desses fatores eleva os riscos de incapacidades, reações adversas e prejuízos nas atividades de trabalho e lazer, gerando déficit na qualidade de vida e declínio no quadro psicológico e comportamental, o que pode induzir a medicalização com mais psicotrópicos ${ }^{30}$.

Apenas nos adultos foi constatada maior prevalência do uso de psicotrópicos nos fumantes. O maior consumo de tabaco entre os indivíduos mais jovens e a estreita relação entre tabagismo e os $\mathrm{TMC}^{13}$, com destaque para os transtornos de ansiedade e uso abusivo de outras substâncias psicoativas ${ }^{31}$, comorbidades mais prevalentes dentre os adultos, expressam a associação entre psicofármacos e o fumo nesses indivíduos. Adiciona-se aos fatos expostos, o prazer e o bem-estar promovido pelo uso da nicotina, que pode se traduzir no apoio às dificuldades no processo de adoecimento, principalmente em relação aos sintomas e enfrentamentos dos estigmas sociais nos TMC, e a tentativa de compensação dos efeitos 
colaterais no uso dos próprios psicotrópicos usados no tratamento ${ }^{32}$.

O uso abusivo de álcool está inversamente associado ao uso de psicotrópicos nos adultos. Este achado pode estar relacionado a adesão quanto a contraindicação no momento da prescrição do uso potencialmente perigoso entre álcool e medicamentos psicotrópicos ${ }^{13}$.

Entre as classes de medicamentos avaliadas neste estudo, os medicamentos mais utilizados pelos adultos foram os antidepressivos. Já nos idosos, a classe mais prevalente quanto ao uso foram os ansiolíticos. O uso de antipsicóticos e estabilizadores de humor foi mais frequente entre os sujeitos com idade $<$ de 60 anos, sendo mais comum entre os mais jovens. Tal informação confirma a maior prevalência de transtornos psicóticos e transtorno bipolar entre os mais jovens, bem como a menor sobrevida entre os acometidos por estes $\mathrm{TMC}^{33}$.

O maior uso de antidepressivos em adultos com idade inferior a 60 anos em comparação aos idosos tem sido reportado na literatura ${ }^{9,34}$. Estudos retratam os riscos de suicídio em adultos jovens em uso de antidepressivos, fato que estimulou as agências regulatórias estadunidense e europeias de medicamentos a emitirem alertas aos prescritores e aos usuários sobre os riscos relacionados ao uso destes fármacos ${ }^{35}$. Esse fato alerta para a necessidade de atenção redobrada no uso racional desses medicamentos nos adultos brasileiros.

Resultados de estudos realizados na cidade de São Paulo ${ }^{21}$ retratam a maior prevalência de uso de antidepressivos em idosos, contrastando com os dados apresentados neste estudo. Dados de inquéritos populacionais realizados em outros países também demonstram situação semelhante do aumento do uso de ansiolíticos com o avançar da idade ${ }^{9,34}$.

De acordo com os critérios estabelecidos no Consenso Brasileiros de Medicamentos Potencialmente Inadequados para idosos ${ }^{36}$, os benzodiazepínicos devem ser evitados naqueles de maior idade, independente das suas condições clínicas, por aumentar o risco de alterações cognitivas, risco de quedas, fraturas e acidentes automobilísticos. A elevada prevalência de benzodiazepínicos está relacionada a busca de alívio dos problemas sociais e controle do estresse da longevidade ${ }^{37}$, representando uma nova forma de subjetivação onde o discurso psicopatológico assume feições decididamente biológica e psicofarmacológica para superar o mal-estar dos dramas sociais e da velhice ${ }^{7}$.
Quanto a fonte de obtenção dos psicotrópicos, iniquidades foram constatadas. Minoria dos usuários desses medicamentos obtém todo seu tratamento de forma gratuita, diferença importante se comparada a estudos com outras classes de medicamentos destinados ao tratamento de doenças crônicas ${ }^{38,39}$. A ampliação do acesso a medicamentos para as DCNT necessita, dentre outros fatores, de uma política de financiamento adequada com estratégias para o aumento da oferta. No Brasil, a disponibilidade de tratamento no SUS para as DCNT é realizada na rede pública e, como estratégia complementar, por meio de copagamento no Programa Farmácia Popular do Brasil (PFPB), que inicialmente, garantiu a distribuição de medicamentos a partir da implantação de uma rede própria de farmácias estatais ${ }^{40}$. Em 2011, com a ampliação da modalidade "Aqui tem Farmácia Popular", em parceria com o comércio varejista, ampliou-se o acesso ao PFPB em todo o país, incluindo isenção para um conjunto de medicamentos, a partir da estratégia denominada "Saúde Não Tem Preço" (SNTP) ${ }^{40}$. No entanto, o elenco disponibilizado nessa última modalidade é mais restrito, não incluindo os psicotrópicos. É razoável considerar que a ausência de estratégias como o SNTP para obtenção dos psicotrópicos, pode impactar no acesso gratuito aos medicamentos.

A prevalência na obtenção de medicamentos pelo SUS é influenciada por variáveis sociais e demográficas. A região de residência apenas influencia o tipo de fonte de obtenção para os idosos, sendo os residentes da região Nordeste os que menos obtém a totalidade do seu tratamento exclusivamente por meio do SUS. Estudo de Matta et al..$^{40}$ aponta que esta região do país possui um dos maiores percentuais de usuários com cuidados exclusivos no SUS (que não possuem plano de saúde) e aquisição de medicamentos em farmácias privadas, indicando deficiências na disponibilidade dos medicamentos no Sistema Público de Saúde. No presente estudo, entre os idosos, observaram-se diferenças na obtenção de tratamento gratuito para os que não possuem plano de saúde.

$\mathrm{Na}$ população adulta, a classe econômica e posse de plano de saúde influenciam a obtenção de todo o tratamento medicamentoso no SUS. Os mais pobres e aqueles com utilização exclusiva do SUS obtiveram seus medicamentos em maior proporção nas farmácias públicas. No entanto, este percentual não alcança metade da população. A obtenção de medicamentos por meio do setor privado ou do sistema de copagamento, por 
usuários com prescrição do SUS, pressupõe um maior comprometimento do orçamento familiar nos gastos com saúde, penalizando principalmente os de menor renda. Situação relacionada, principalmente, a dificuldade de encontrar todos os medicamentos que foram indicados para o tratamento nas farmácias do SUS ${ }^{40}$.

Os transtornos mentais estão entre as doenças crônicas que causam maior incapacidade. Comorbidades como a esquizofrenia, transtorno bipolar, episódios depressivos graves ou no controle de ataques de pânico têm o tratamento medicamentoso como opção terapêutica preferencial $^{26}$. A descontinuidade do tratamento ou a não obtenção pela dificuldade de desembolso para o custeio do medicamento pode ocasionar uma maior carga de incapacidade temporária ou permanente aos indivíduos, levando a perda de produtividade e prejuízos no âmbito familiar ${ }^{41}$.

O financiamento público de medicamentos no Brasil é de responsabilidade da União, estados e municípios, sendo a Relação Nacional de Medicamentos Essenciais (Rename) a lista oficial que norteia a seleção, a oferta nos serviços de saúde, a prescrição e a dispensação de medicamentos financiados no SUS ${ }^{42}$. Os grupos de medicamentos antidepressivos, estabilizadores de humor, ansiolíticos e antipsicóticos constam entre os medicamentos que podem ser ofertados na rede de serviços desde a Atenção Básica ao nível especializado de Atenção à Saúde.

A ampliação da obtenção gratuita do tratamento, evidenciada para aqueles de menor renda e nos que dependem de atenção à saúde exclusiva no SUS, reflete a magnitude da importância de ampliação das estratégias de equidade, inclusive nas Políticas Farmacêuticas, para redução das desigualdades no acesso gratuito de medicamentos.

Como limitações, por ser um estudo transversal, não foi possível identificar a relação en- tre causa e efeito, como por exemplo, na relação entre autoavaliação de saúde e o uso de psicotrópicos. Além disso, deve-se considerar que as informações foram autorreferidas, e o possível viés de memória, principalmente nos relatos das indicações dos medicamentos, nas consultas com profissionais de saúde, e quanto à correta identificação da forma de obtenção dos medicamentos.

\section{Considerações finais}

Este estudo apresenta dados inéditos sobre a prevalência nacional da utilização de psicotrópicos e sua distribuição na população adulta e idosa brasileira, bem como o perfil dos usuários e as fontes de obtenção desses medicamentos. Para o conjunto dos adultos que referiram uso de psicotrópicos, maior parcela os obteve com recursos próprios. Para aqueles que obtiveram esses medicamentos no SUS, maiores proporções foram observadas pelos mais pobres e nos que não possuíam plano de saúde, sugerindo que as estratégias de equidade estabelecidas para o enfrentamento das desigualdades no acesso a medicamentos podem ter tido algum alcance em relação aos psicotrópicos.

No entanto, a baixa proporção de obtenção dos psicotrópicos no SUS aponta para a necessidade de estudos futuros sobre a disponibilidade destes medicamentos nos serviços e a conformidade entre os medicamentos prescritos nos serviços públicos de saúde e aqueles oferecidos na Relação Nacional de Medicamentos Essenciais. Os dados deste estudo poderão contribuir para o planejamento e gestão das políticas farmacêuticas e, em especial, para a estruturação de cuidados e tratamento na rede de atenção psicossocial do país, visando melhorar a qualidade de vida dos usuários desses medicamentos e garantindo o direito à saúde a essa população.

\section{Colaboradores}

PS Rodrigues, PMSB Francisco e KS Costa participaram da concepção, execução das análises, redação e aprovação da versão final do manuscrito. AT Fontanella e RB Borges colaboraram na revisão das análises dos dados e aprovação final do manuscrito. 


\section{Agradecimentos}

Ao Ministério da Saúde por meio da Secretaria de Ciência e Tecnologia e Insumos Estratégicos (SCTIE/MS) pelo financiamento e apoio técnico para a realização da Pesquisa Nacional sobre Acesso, Utilização e Promoção do Uso Racional de Medicamentos e, em especial, à equipe de suporte do projeto, nos nomes de Amanda Ramalho da Silva e Luciano Santos Pinto Guimarães.

À Coordenação de Aperfeiçoamento de Pessoal de Nível Superior (CAPES), pela bolsa de doutorado concedida a Patrícia Silveira Rodrigues.

\section{Referências}

1. Mendes EV. O cuidado das condições crônicas na atenção primária à saúde: o imperativo da consolidação da estratégia da saúde da família. Brasília: OPAS; 2012.

2. Veras R. Envelhecimento populacional contemporâneo: demandas, desafios e inovações. Rev Saude Publica 2009; 43(3):548-554.

3. Bonadiman CSC, Passos VMA, Mooney M, Naghavi M, Melo APS. A carga dos transtornos mentais e decorrentes do uso de substâncias psicoativas no Brasil: Estudo de Carga Global de Doença, 1990 e 2015. Rev Bras Epidemiol 2017; 20(1):191-204.

4. GBD 2016 Disease and Injury Incidence and Prevalence Collaborators. Global, regional, and national incidence, prevalence, and years lived with disability for 328 diseases and injuries for 195 countries, 19902016: a systematic analysis for the Global Burden of Disease Study 2016. Lancet 2017; 390(10100):12111259.

5. Kapp PA, Klop AC, Jenkins LS. Drug interactions in primary health care in the George subdistrict, South Africa: a cross-sectional study. South African Fam Pract 2013; 55(1):78-84.

6. Mark TL. For what diagnoses are psychotropic medications being prescribed? CNS Drugs 2010; 24(4):319326.

7. Birman J. Mal-estar na atualidade: a psicanálise e as novas formas de subjetivação. $5^{\mathrm{a}}$ ed. Rio de Janeiro: Civilização Bras; 2005.

8. Mengue SS, Bertoldi AD, Boing AC, Tavares NUL, Dal Pizzol TS, Oliveira MA, Arrais PSD, Ramos LR, Farias MR Luiza VL, Bernal RTI, Barros AJD. National Survey on Access, Use and Promotion of Rational Use of Medicines (PNAUM): household survey component methods. Rev Saude Publica 2016; 50(Supl. 2):4s.

9. Beck CA, Williams JV, Wang JL, Kassam A, El-Guebaly N, Currie SR, Maxwell CJ, Patten SB. Psychotropic Medication Use in Canada. Can J Psychiatry 2005; 50(10):605-613.

10. Paulose-Ram R, Safran MA, Jonas BS, Gu Q, Orwig D. Trends in psychotropic medication use among U.S. adults. Pharmacoepidemiol Drug Saf 2007; 16(5):560570 .

11. Ohayon MM, Lader MH. Use of psychotropic medication in the general population of France, Germany, Italy, and the United Kingdom. J Clin Psychiatry 2002; 63(9):817-825.

12. Rojas G, Fritsch R, Gaete J, González I, Araya R. Use of psychotropic medication in Santiago, Chile. J Ment Heal 2005; 14(4):407-414.

13. Carrasco-Garrido P, Hernández-Barrera V, JiménezTrujillo I, Esteban-Hernández J, Álvaro-Meca A, López-de Andrés A, DelBarrio-Fernández JL, JiménezGarcía R. Time trend in psychotropic medication use in Spain: a nationwide population-based study. Int J Environ Res Public Health 2016; 13(12):1177.

14. Prado MAMB, Francisco PMSB, Barros MBA. Uso de medicamentos psicotrópicos em adultos e idosos residentes em Campinas, São Paulo: um estudo transversal de base populacional. Epidemiol Serviços Saúde 2017; 26(4):747-758. 
15. Neutel CI, Walop W. Drug Utilization by Men and Women: Why the Differences? Drug Inf J 2005, 39(3):299-310.

16. Giovanella L, Escorel S, Lobato LVC, Noronha JC, Carvalho AI, organizadores. Politicas e sistema de saúde no Brasil. Rio de Janeiro: Editora Fiocruz; 2012.

17. Viana MC, Silveira CM, Andrade LHSG. Epidemiologia dos transtornos mentais da mulher. In: Rennó Jr J, Ribeiro HL, organizadores. Tratado de saúde mental da mulher. São Paulo: Atheneu; 2012.

18. Gomes ALC, Nagashima AMS. O feminino Aprisionado, Patologizado e Medicalizado: Impactos na Saúde Mental das Mulheres. In: Amarante P, Pitta AMFP, Oliveira WF, organizadores. Patologização e Medicalização da Vida. São Paulo: Zagodoni; 2018.

19. Grinshpoon A, Marom E, Weizman A, Ponizovsky AM. Psychotropic drug use in Israel: results from the national health survey. Prim Care Companion J Clin Psychiatry 2007; 9(5):356-363.

20. Alonso J, Angermeyer MC, Bernert S, Bruffaerts R, Brugha TS, Bryson H, Girolamo G, Graaf R, Demyttenaere K, Gasquet I, Haro JM, Katz SJ, Kessler RC, Kovess V, Lépine JP, Ormel J, Polidori G, Russo LJ, Vilagut G, Almansa J, Arbabzadeh-Bouchez S, Autonell J, Bernal M, Buist-Bouwman MA, Codony M, Domingo-Salvany A, Ferrer M, Joo SS, Martínez-Alonso M, Matschinger H, Mazzi F, Morgan Z, Morosini P, Palacín C, Romera B, Taub N, Vollebergh WAM, ESEMeD/MHEDEA 2000 Investigators, European Study of the Epidemiology of Mental Disorders (ESEMeD) Project. Psychotropic drug utilization in Europe: results from the European Study of the Epidemiology of Mental Disorders (ESEMeD) project. Acta Psychiatr Scand Suppl 2004; (420):55-64.

21. Noia AS, Secoli SR, Duarte YAO, Lebrão ML, Lieber NSR. Fatores associados ao uso de psicotrópicos por idosos residentes no Município de São Paulo. Rev da Esc Enferm da USP 2012; 46(esp.):38-43.

22. Rodrigues MAP, Facchini LA, Lima MS. Modificações nos padrões de consumo de psicofármacos em localidade do Sul do Brasil. Rev Saude Publica 2006; 40(1):107-114.

23. Gaskin DJ, Briesacher BA, Limcangco R, Brigantti BL. Exploring racial and ethnic disparities in prescription drug spending and use among Medicare beneficiaries. Am J Geriatr Pharmacother 2006; 4(2):96-111.

24. Gary FA. Stigma: Barrier to mental health care among ethnic minorities. Issues Ment Health Nurs 2005; 26(10):979-999.

25. Alves AAM, Rodrigues NFR. Determinantes sociais e económicos da Saúde Mental. Rev Port Saúde Pública 2010; 28(2):127-131.

26. Cordioli AV. Psicofármacos nos transtornos mentais. Porto Alegre: Universidade Federal do Rio Grande do Sul; 2005.

27. Brasil. Agência Nacional de Vigilância Sanitária (ANVISA). Portaria n ${ }^{\circ}$ 344, de 12 de maio de 1998: Aprova o regulamento técnico sobre substâncias e medicamentos sujeitos a controle especial. Diário Oficial da União; 1998.
28. Viegas MA, Kenya Noronha KVMS, Menezes RM, Souza MN, Reis CB, Martins DR, Gomes L. Desigualdade socioeconômica no acesso aos serviços de saúde no Brasil: um estudo comparativo entre as regiões brasileiras em 1998 e 2008. Econ Apl 2013; 17(4):623645.

29. Garnelo L, Sousa ABL, Silva CO. Regionalização em Saúde no Amazonas: avanços e desafios. Cien Saude Colet 2017; 22(4):1225-1234.

30. Organização Pan-Americana da Saúde (OPAS). A atenção à saúde coordenada pela APS: construindo as redes de atenção no SUS: contribuições para o debate. Brasília: OPAS; 2011.

31. Breslau N, Novak SP, Kessler RC. Psychiatric disorders and stages of smoking. Biol Psychiatry 2004; 55(1):6976.

32. Rondina RC, Gorayeb R, Botelho C. Relação entre tabagismo e transtornos psiquiátricos. Rev Psiquiatria Clin 2003; 30(6):221-228.

33. Charlson FJ, Baxter AJ, Dua T, Degenhardt L, Whiteford HA, Vos T. Excess mortality from mental, neurological and substance use disorders in the Global Burden of Disease Study 2010. Epidemiol Psychiatr Sci 2015; 24(2):121-140.

34. Goldney R, Bain M. Prevalence of Psychotropic Use in a South Australian Population. Australas Psychiatry 2006; 14(4):379-383.

35. Mitchell AM, Davies MA, Cassesse C, Curran R. Antidepressant Use in Children, Adolescents, and Young Adults: 10 Years After the Food and Drug Administration Black Box Warning. J Nurse Pract 2014; 10(3):149-156

36. Oliveira MG, Amorim WW, Oliveira CRB, Coqueiro HL, Gusmão LC, Passos LC. Consenso brasileiro de medicamentos potencialmente inapropriados para idosos. Geriatr Gerontol Aging 2016; 10(4):168-181.

37. Alvarenga JM, Giacomin KC, Loyola Filho AI, Uchoa E, Firmo JOA. Uso crônico de benzodiazepínicos entre idosos. Rev Saude Publica 2014; 48(6):866-872.

38. Mengue SS, Bertoldi AD, Ramos LR, Farias MR, Oliveira MA, Tavares NUL, Arrais PSD, Luiza VL, Dal Pizzol TS. Access to and use of high blood pressure medications in Brazil. Rev Saude Publica 2016; 50(Supl. 2):8s.

39. Meiners MMMA, Tavares NUL, Guimarães LSP, Bertoldi AD, Dal Pizzol TS, Luiza VL, Mengue SS, Merchan-Hamann E. Access and adherence to medication among people with diabetes in Brazil: evidences from PNAUM. Rev Bras Epidemiol 2017; 20(3):445-459.

40. Matta SR, Bertoldi AD, Emmerick ICM, Fontanella AT, Costa KS, Luiza VL. Fontes de obtenção de medicamentos por pacientes diagnosticados com doenças crônicas, usuários do Sistema Único de Saúde. Cad Saude Publica 2018; 34(3):e00073817.

41. Razzouk D. Economia da saúde aplicada à saúde mental. In: Mateus MD, organizador. Políticas de saúde mental. São Paulo: Instituto de Saúde; 2013. p. 231251. 
42. Brasil. Ministério da Saúde (MS). Secretaria de Ciência Tecnologia e Insumos Estratégicos. Departamento de Assistência Farmacêutica e Insumos Estratégicos. Relação Nacional de Medicamentos Essenciais: RENAME 2017. Brasília: MS; 2017.

Artigo apresentado em 10/07/2018

Aprovado em 22/02/2019

Versão final apresentada em 24/02/2019 